\title{
Spectral Methods in Gravitation and Cosmology
}

\section{H. P. de Oliveira*}

Instituto de Física, Universidade do Estado do Rio de Janeiro

R. São Francisco Xavier, 524, Rio de Janeiro, 20550-013, RJ, Brazil.

E-mail: oliveira@dft.if.uerj.br

\section{J. E. Skea}

Instituto de Física, Universidade do Estado do Rio de Janeiro

R. São Francisco Xavier, 524, Rio de Janeiro, 20550-013, RJ, Brazil.

E-mail: jmskedft.if.uerj.br

\section{O. C. Castellani}

Centro Brasileiro de Pesquisas Físicas

Rua Dr. Xavier Sigaud 150, Rio de Janeiro, RJ, 22290-180, Brazil

E-mail: castellani@cbpf.br

The interest in applying spectral methods to problems of gravitation and cosmology has increased considerably. In this paper we briefly describe the main aspects of the spectral methods focusing on the Galerkin method. We have discussed in the sequence the problem of the spherical gravitational collapse of a massless scalar field from a dynamical system approach provided by the Galerkin method.

Fifth International Conference on Mathematical Methods in Physics - IC2006

April 24-28 2006

Centro Brasilerio de Pesquisas Fisicas, Rio de Janeiro, Brazil

\footnotetext{
* Speaker.
} 


\section{Introduction}

The General Theory of Relativity (GR) constitutes one of the major scientific achievements of Science, and so far it has passed quite well in all experimental tests. Basically, GR establishes a system of ten nonlinear and coupled partial differential equations that governs the dynamics of the gravitational field represented by the symmetric tensor $g_{\alpha \beta}, \alpha, \beta=0,1,2,3$. The nonlinear nature of the field equations is the main obstacle to obtain exact solutions of the field equations unless, of course, perturbative approaches are assumed so that the equations become linear, or under the assumption of idealized symmetries. Therefore, in order to study the dynamics of the gravitational field in more general situations the use of numerical techniques seems to be the only possible strategy to circumvent the difficulty posed by the nonlinearities of the field equations. Numerical relativity has become a very fertile and at the same time challenging field of research as recently covered by several interesting reviews[1], where the improvement of specific numerical techniques adapted to relativistic problems along with the growth of computational resources figure as the factors for the advance of numerical relativity. However, the complete understanding of important problems in relativistic astrophysics such as non-spherical collapse and nonlinear regimes of emission of gravitational waves is still not complete.

A promising approach in treating numerically nonlinear problems is provided by the so-called spectral methods $[2,3,4]$. The spectral methods adopt a distinct strategy if compared with the finite difference scheme. For instance, considering a function $u(t, x)$ satisfying a given one dimensional partial differential equation, it will be approximate as a series of the type $u_{a}(t, x)=\sum_{k=0}^{N} a_{k}(t) \psi_{k}(t)$, where the basis or trial functions $\psi_{k}(x)$ are known analytical polynomials such as Fourier, Legendre, Chebyshev, etc. In general, by increasing the truncation order $N, u_{a}(t, x)$ approaches of the exact solution of the problem. There are distinct types of spectral methods among which we list the Galerkin method[5], the collocation method and the Tau-method. These methods have an attractive feature which is to transform any partial differential equation into a finite set of ordinary differential equations, or simply a dynamical system whose dimension is dictated by the truncation order $N$. Another important robust feature is the high accuracy achieved with a small truncation order, or equivalently using a moderate or low computational resources. The combination of such features can be understood as the low dimensional dynamical system approach broadly applied in problems of turbulence of fluids[6]. On the other hand, applications of this approach in specific problems of Cosmology[7] and Gravitation[2, 3, 8, 9, 10, 11] has been done recently.

In this paper our objective is to apply the Galerkin method to the problem of critical phenomena in gravitational collapse of a scalar field[12]. As a matter of fact, critical phenomena in gravitational collapse has opened a new venue of investigation of the strong gravitational field regime, and has been investigated intensively until now. Choptuik originally studied the spherically symmetric collapse of massless scalar field and found a series of intriguing new features typical of a system described in statistical physics under phase transition. The original results refer to the collapse of initial data families of massless scalar field whose strength is characterized be some parameter $p$. Essentially, there exists a critical value $p^{*}$ such that the solution of the field equations are divided into three classes: (i) the subcritical solutions for which $p<p^{*}$, and the collapsing matter eventually disperses leaving behind flat spacetime; (ii) critical solutions that have $p=p^{*}$ 
and exhibits self-similar echoing in the neighborhood of a central singularity; and (iii) supercritical solutions characterized by $p>p^{*}$, and the scalar field collapses to form a black hole. In particular for marginally supercritical evolutions, the masses of the black holes obey the following scaling law

$$
M_{B H} \propto\left|p-p^{*}\right|^{\gamma}
$$

where $\gamma \approx 0.37$ is independent of the initial data. Several authors have recovered these properties considering the collapse of distinct matter field and symmetries[13], but there still are some open questions such as the generality of critical phenomena, or the underlying physics responsible for the amazing similarity with the usual critical phenomena described in Statistical Mechanics.

The paper is organized as follows. In Section 2 the basic equations describing a self gravitating spherically symmetric scalar field are exhibited. The linearized field equations are presented in Section 3 as a test for the Galekrin method, where the approximate and exact solutions are compared. Sections 4 and 5 are devoted to the application of the Galerkin method to the full nonlinear field equations; the dynamical system is obtained for several truncation orders along with the presentation of the numerical results. Finally, in Section 6 we conclude. Thorough the paper we assume $8 \pi G=c=1$.

\section{The basic equations}

We consider the general spherically symmetric line element written as

$$
d s^{2}=-\mathrm{e}^{2 \beta} \frac{V}{r} d u^{2}-2 \mathrm{e}^{2 \beta} d u d r+r^{2} d \Omega^{2},
$$

where $\beta=\beta(u, r), V=V(u, r)$ and $d \Omega^{2}=d \theta^{2}+\sin \theta^{2} d \varphi^{2} ; u$ is the usual retarded null coordinate. The massless scalar field $\phi=\phi(u, r)$ is the only source of curvature, such that the relevant field equations are

$$
\begin{aligned}
\beta_{, r} & =\frac{r}{4} \phi_{, r}^{2} \\
V_{, r} & =\mathrm{e}^{2 \beta} \\
r \phi_{, u r}+\phi_{, u} & =\frac{1}{2 r}\left(r V \phi_{, r}\right)_{, r} .
\end{aligned}
$$

The first two equations are constraint equations relating the metric functions $\beta(u, r)$ and $V(u, r)$ with the scalar field; the third is the Klein-Gordon equation. This equation allows to evolve the scalar field once the initial data $\phi\left(u_{0}, r\right)$ specified at the initial null hypersurface $u=u_{0}$.

As we are going to see, the boundary conditions are of fundamental importance for the implementation of the Galerkin method. Accordingly, assuming the asymptotically flat spacetimes, it must be guaranteed that

$$
\phi \rightarrow 0, \beta \rightarrow 0, V \rightarrow r
$$

at the spatial infinity $r \rightarrow \infty$. In this case, the scalar field has the asymptotic expansion 


$$
\phi=\frac{Q(u)}{r}+\frac{Q_{2}(u)}{r^{2}}+\ldots
$$

where $Q(u)$ is the scalar monopole and $Q_{2}(u)$ is the Newman-Penrose constant for the scalar field[14]. The boundary conditions for the metric functions at the origin $r=0$ are expressed as follows

$$
\beta \simeq \mathscr{O}\left(r^{2}\right), V \simeq r+\mathscr{O}\left(r^{3}\right),
$$

together with the assumption of $\phi$ being finite at the origin. These are the Bondi conditions for the regularity of the spacetime at the origin. Also, $\mathrm{e}^{2 \beta}=V / r=1$ at $r=0$, corresponds to select $u$ as the proper time at $r=0$ central world line.

Another important quantity to be introduced is the mass function, $m(u, r)$,

$$
1-\frac{2 m\left(x^{\alpha}\right)}{r} \equiv g^{\mu v} r_{, \mu} r_{, v}=\frac{V \mathrm{e}^{2 \beta}}{r}
$$

which is interpreted as the effective gravitational mass inside the 2-sphere of radius $r$, and agrees with the Bondi and ADM masses in the asymptotic flat spacetimes.

For the sake of convenience we follow Goméz and Winicour[15] and introduce the auxiliary field $\Phi(u, r)$ defined as

$$
\Phi \equiv r \phi
$$

The boundary conditions are $\Phi(u, 0)=0$ and $\Phi(u, \infty)=Q(u)$.

\section{The weak field limit: linear theory}

We begin studying the linearized field equations or the evolution of weak fields. In this approximation Eqs. (2.2) and (2.3) yield $\beta \approx 0$ and $V \approx r$ respectively, therefore reducing the problem to the dynamics of the Klein-Gordon equation in Minkowski spacetime. In terms of the auxiliary field $\Phi(u, r)$, the evolution equation has the form

$$
2 \Phi_{, u r}-\Phi_{, r r}=0
$$

whose general exact solution that satisfies the boundary condition at the origin is

$$
\Phi_{\text {exact }}=F(u+2 r)-F(u),
$$

where $F$ is an arbitrary function. The above exact solution will be used to test the Galerkin method as described below.

The cornerstone of the Galerkin method is the choice of a set of basis functions, or trial functions, for which the the field $\Phi$ is approximated in the following way

$$
\Phi(u, r)=\sum_{k=0}^{N} a_{k}(u) \psi_{k}(r),
$$


where $a_{k}(u)$ are the unknown modal coefficients and $N$ is the order of truncation; $\psi_{k}(r), k=$ $0,1, . ., N$ are known trial functions defined in the interval $0 \leq r<\infty$. By an appropriate choice of the trial functions the convergence of the decomposition (3.3) to the exact $\Phi$ is guaranteed as $N$ is made arbitrarily large. As demanded by the Galerkin prescription the trial functions must satisfy the boundary conditions imposed for $\Phi(u, r)$, namely, $\psi_{k}(0)=0$ and $\psi_{k}(\infty)$ being finite for any $k$.

The next step is to substitute the decomposition (3.3) into the Klein-Gordon equation yielding what is known as the residual equation:

$$
\operatorname{Res}(u, r)=2 \sum_{k=0}^{N} \dot{a}_{k}(u) \psi_{k}(r)-\sum_{k=0}^{N} a_{k}(u) \psi_{k}^{\prime \prime}(r) .
$$

Here dot and prime means derivative with respect to $u$ and $r$, respectively. Next we project the residual equation into each trial function $\psi_{n}(r), n=0,1, . ., N$, which is in general defined as

$$
\left\langle\operatorname{Res}(u, r), \psi_{n}(r)\right\rangle=\int_{0}^{\infty} \operatorname{Res}(u, r) \psi_{n}(r) w(r) d r .
$$

where $w(r)$ is the weight function associated to the selected basis the trial functions. According to the Galerkin method the wave equation (3.1) is reduced to a finite set of ordinary differential equations for the modal coefficients $a_{k}(u)$ by imposing

$$
\left\langle\operatorname{Res}(u, r), \psi_{n}(r)\right\rangle=0,
$$

for all $n=0,1, . ., N$. In general these equations can be cast in the following form

$$
\dot{a}_{j}(u)=\mathscr{F}_{j}\left(a_{0}, a_{1}, . ., a_{N}\right), j=0,1, . ., N,
$$

where $\mathscr{F}_{j}\left(a_{0}, a_{1}, . ., a_{N}\right)$ are linear functions of the modal coefficients.

The set of trial functions $\psi_{k}(r)$ must satisfy the boundary conditions imposed for the field $\Phi(u, r)$. In this vein, the following combination of rational Chebyshev functions[16], $T L_{k}(r)$, was chosen

$$
\psi_{k}(r)=T L_{k+1}(r)+T L_{k}(r) .
$$

The rational Chebyshev functions are defined in the semi-infinite interval ${ }^{1}$, and the weight function is given by $w(r)=r^{-1 / 2} /(1+r)$. Notice that by construction $\psi_{k}(0)=0$ for any $k$, and the scalar charge is now given by

$$
Q(u)=\sum_{k=0}^{N} 2 a_{k}(u),
$$

providing the correct asymptotic form of the scalar field at the spatial infinity according to Eq. (2.6).

We are now in conditions of confronting the exact solution for the auxiliary field $\Phi(u, r)$ with its evolution computed using the Galerkin method. The starting point is to select the initial profile

\footnotetext{
${ }^{1}$ The rational Chebyshev functions are obtained using the transformation $x=(r-1) /(r+1)$, where $-1 \leq x \leq 1$ and $0 \leq r<\infty$
} 
$\Phi\left(u_{0}, r\right)=\Phi_{0}(r)$ that determines the initial values for the modal coefficients through the decomposition $\Phi_{0}(r) \equiv \sum_{k=0}^{N} a_{k}\left(u_{0}\right) \psi_{k}(r)$. Two examples of the one parameter set of initial data will be considered[15]:

$$
\text { (i) } \Phi_{0}(r)=\lambda r /(1+r), \quad(i i) \Phi_{0}(r)=\lambda \tanh (r) \text {, }
$$

where $\lambda$ is the initial scalar charge of the distribution. Once the initial conditions are specified the evolution of each modal coefficient is determined from the system (3.7), and, as a consequence, the overall dynamics of $\Phi(u, r)$ through the decomposition (3.3) is known. In particular as a consequence of the first initial data the modal coefficients are given by $a_{k}(0)=0, k=1,2, \ldots, N$ and $a_{0}(0)=\lambda / 2$, producing an exact fitting. In Fig. 1(a) we illustrate the evolution of the modal coefficients $a_{1}, a_{2}, . ., a_{5}$ for $N=5$. Since the first initial data is taken into account their initial values are all zero, moreover it can be noticed that at each instant $a_{1}>a_{2}>\ldots>a_{5}$, implying in the convergence of the Galerkin decomposition. The same aspect is verified if $N$ is increased.

In order to provide a quantitative measure of the error between the approximate solution corresponding to a given truncation order $N$ and the exact solution we evaluate the $L_{2}$-error, or the rms error, defined as

$$
L_{2}=\sqrt{\int_{0}^{1}\left[\Phi_{\text {exact }}(u, x)-\sum_{k=0}^{N} a_{k}(u) \psi_{k}(x)\right]^{2} d x}
$$

Notice that a new radial coordinate $x$ was introduced via $r \rightarrow x=r /(1+r)$ to compactify the spatial domain. By plotting the rms error at each instant for both initial data families we can envisage that a satisfactory accuracy is achieved even if the truncation order is relatively low. Also, the effect of increasing the truncation order $N$ demonstrates the rapid convergence of the Galerkin decomposition (3.3). In Fig. 1(b) we have depicted the evolution of the error considering the second initial data family.
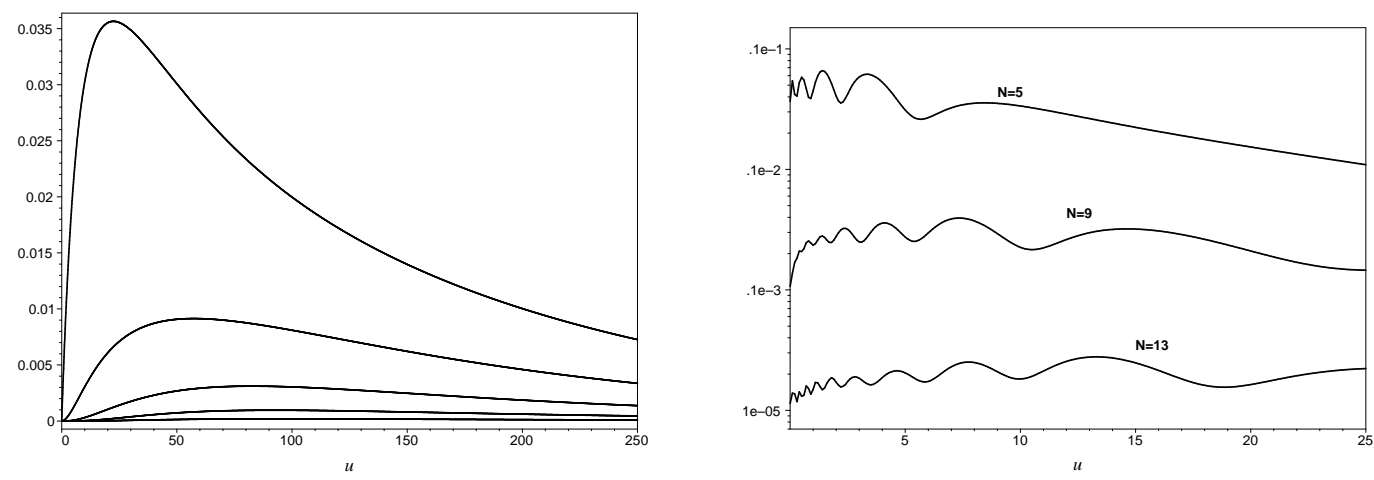

Figure 1: (a) Evolution of the modal coefficients $a_{1}, a_{2}, \ldots, a_{5}$ (upper to lower curves) for the first initial data family with $\lambda=1$. (b) $L_{2}$-error evaluated at each instant for distinct truncation orders, where it is clear the convergence as $N$ is increased. In these experiments we have considered the second initial data family. 


\section{The nonlinear case}

In order to apply the Galerkin method to the system formed by Eqs. (2.2), (2.3) and (2.4) the same procedure as outlined in the last Section will be performed. We assume the decomposition (3.3) for the auxiliar field $\Phi(u, r)$ as the fundamental piece to implement the Galerkin method, and therefore the dynamical system for the modal coefficients. The metric function $\beta(u, r)$ can determined after integrating Eq. (2.2) as

$$
\beta(u, r)=\frac{1}{4} \int_{0}^{r} r\left[\sum_{k=0}^{N} a_{k}(u)\left(\frac{\psi_{k}(r)}{r}\right)^{\prime}\right]^{2} d r .
$$

This integral can be evaluated exactly for any truncation order $N$ resulting in an expression which is quadratic with respect to the modal coefficients. Next, the function $V(u, r)$ is obtained from (2.3) through

$$
V(u, r)=\int_{0}^{r} \mathrm{e}^{2 \beta(u, r)} d r
$$

To integrate this expression in a closed form an additional approximation is assumed. The integrand may be expanded as $\exp (2 \beta)=1+2 \beta+2 \beta^{2}+. . \simeq 1+\sum_{k=1}^{J}(2 \beta)^{k} / k$ !. For instance, the linear approximation studied previously is equivalent to $\exp (\beta) \approx 1$, which implies $V(u, r) \approx r$. The next approximation, $\exp (2 \beta) \approx 1+2 \beta$, will be named quasilinear, since despite being linear in $\beta$ is quadratic in the modal coefficients. In obtaining the dynamical system for the modal coefficients we shall take into account the effect of increasing the truncation order $N$ as well as $J$. Using this procedure it will be instructive to exhibit the expressions for $\beta(u, r)$ and $V(u, r)$ near the origin, or

$$
\beta(u, r)=\left(\frac{1}{2} a_{0}^{2}-7 a_{0} a_{1}+\ldots\right) r^{2}+\ldots, V(u, r)=r+\left(\frac{1}{3} a_{0}^{2}-\frac{14}{3} a_{0} a_{1}+\ldots\right) r^{3}+. .
$$

which is in agreement with the boundary conditions (2.7).

Once a suitable expression for $V(u, r)$ is established, we substitute it into the Klein-Gordon equation (2.4) for the auxiliar variable $\Phi$ along with the decomposition (3.3). In this case, we obtain the following residual equation for the nonlinear problem

$$
\begin{aligned}
& \operatorname{Res}(u, r)=\sum_{k=0}^{N}\left[2 \dot{a}_{k}(u) \psi_{k}^{\prime}(r)-a_{k}(u) \psi_{k}^{\prime \prime}(r)\right]+\left(1-\frac{V}{r}\right) \sum_{k=0}^{N} a_{k}(u) \psi_{k}^{\prime \prime}(r)-\frac{1}{r}\left(\frac{V}{r}\right)^{\prime} \times \\
& \sum_{k=0}^{N}\left[a_{k}(u)\left(r \psi_{k}^{\prime}(r)-\psi_{k}(r)\right)\right] .
\end{aligned}
$$

Note that the first term on the rhs of the residual equation describes the linear wave equation (cf. Eq. (3.4)), while the remaining terms correspond to the nonlinearities. The last step of the Galerkin method consists in to impose that the projection of the residual equation into each basis function $\psi_{n}(r), n=0,1,2, . ., N$ vanishes. These $N+1$ resulting relations can be solved for each $\dot{a}_{n}$ resulting in a dynamical system that has the form 


$$
\dot{a}_{n}(u)=\mathscr{F}_{n}^{N L}\left(a_{0}, a_{1}, . ., a_{N}\right), n=0,1, . ., N,
$$

where $\mathscr{F}_{n}^{N L}$ are nonlinear functions of the modal coefficients. Therefore, Eqs. (2.2)-(2.4) describing the evolution of a scalar field in spherically symmetric spacetimes are reduced to a nonlinear $(N+1)$-dimensional dynamical system in the phase space of the modal coefficients $a_{k}(u)$. To integrate the system (4.5) we need to supply the initial conditions $a_{k}(0)$ evaluated from the initial data $\Phi_{0}(r)=\sum_{k=0}^{N} a_{k}(0) \psi_{k}(r)$. In what follows we have obtained dynamical systems for the quasilinear approach, i. e., $J=1$ and with $N$ varying from 6 to 12 ; also considering $J=2, N$ was made to vary from 3 to 7 .
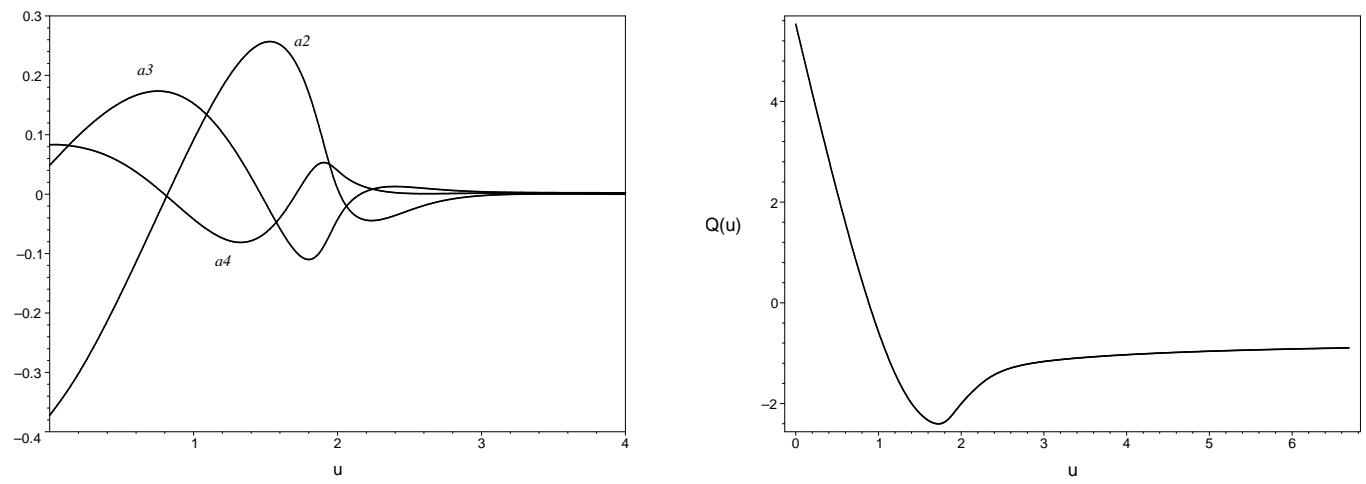

Figure 2: Evolution of the modal coefficients $a_{2}(u), a_{3}(u)$ and $a_{4}(u)$ for the second initial data family for which $\lambda=5.6$ showing that asymptotically all modal coefficients vanish. The behavior of the scalar charge $Q(u)$ also indicates that the asymptotic configuration is the Minkowski spacetime.

The numerical experiments were performed using the initial data families given by (3.10). In both cases the basic features of the dynamics depend on the parameter $\lambda$ which is associated to the initial strength of the scalar field, and not to a particular form of $\Phi_{0}(r)$. For the sake of simplicity in most of the numerical experiments the initial conditions for the modal coefficients will correspond to the first initial data family. Also, our numerical experiments have indicated that the structure of all solutions in phase space seems to be independent of particular choices of $N$ and $J \geq 1$.

The dynamics in the phase space of modal coefficients is described as follows. Basically we have found two main classes of solutions that form two basins of attraction in phase space. The first class corresponds to sufficiently small values of $\lambda$ for which the origin of phase space is a stable critical point, or simply an attractor. Hence, asymptotically $a_{k}(u) \rightarrow 0$ implying that $\exp (2 \beta) \rightarrow 1$, $V \rightarrow r$ and the scalar field approach to zero; or equivalently the Minkowski solution is attained. According to previous studies on gravitational collapse of scalar fields, this set of solutions is known as subcritical. In Fig. 2 we have illustrated such a class of solution depicting the evolution of some modal coefficients and the scalar charge $Q(u)=\lim _{r \rightarrow \infty} \sum a_{k}(u) \psi_{k}(r)$ belonging to a Galerkin decomposition of $\Phi(u, r)$ with $N=9$ and corresponding to the second initial data family. Notice that although some modal coefficients exhibit an initial stage of growth, they eventually tend to zero and, consequently the same outcome is observed for the scalar charge $Q(u)$. 

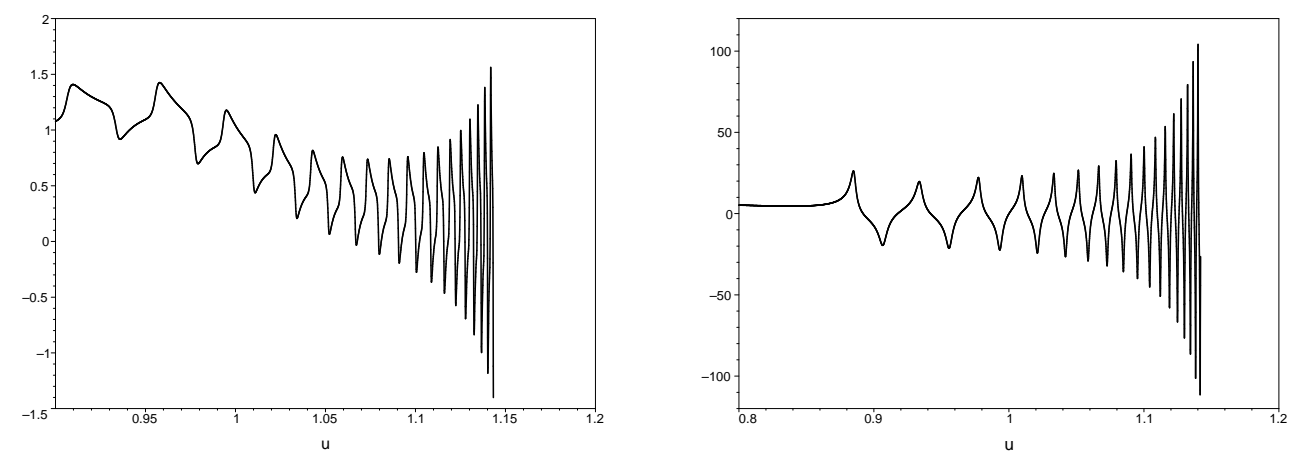

Figure 3: Evolution of $a_{0}(u)$ (left panel) and the scalar field $\phi(u, r=0)$ (right panel). We have chosen $N=9$ and $\lambda=7.3$ for the first initial data family.

On the other hand, for sufficiently large values of $\lambda$ the generated orbit starts to explore regions of phase space not close to the origin, and asymptotically tends to the infinity region in phase space characterized by $\sum_{k=0}^{N} a_{k}^{2}=\infty$. This can be understood as an indication that the scalar field has enough strength to hold the collapse until the formation of a black hole, since it eventually happens that at some point for which $2 m / r=1-V \mathrm{e}^{-2 \beta} / r \rightarrow 1$ which signalizes the formation of an apparent horizon, and therefore a black hole. These solutions are known as supercritical and the black hole formation constitutes the second basin of attraction present in the phase space. The behavior of the first modal coefficient $a_{0}(u)$ and the scalar field $\phi(u, r)=\sum a_{k}(u) \psi_{k}(r) / r$ evaluated at the origin are shown in Fig. 3. Note the oscillatory regime before the orbit escapes to the infinity region in phase space suggesting the presence of a periodic structure intermediate between both basins of attraction.

Probably the most interesting aspect of the dynamics provided by the Galerkin method is the existence of a boundary that separates both subcritical and supercritical classes of solution. This boundary corresponds to a periodic orbit, or a limit cycle, in the modal phase space and obtained by fine tuning the parameter $\lambda$ that, as we have seen, controls the initial strength of the scalar field. The more precise $\lambda$ is adjusted to approach the actual critical value $\lambda_{*}$, the longer is the time in which the modal coefficients oscillate periodically. Eventually, one of the two above described outcomes will be reached since the limit cycle is unstable. It is also worth mentioning that the same limit cycle is attained if the second initial data family is taken into account, suggesting that this structure is unique in phase space. In Fig. 4(b) the evolution the modal coefficient $a_{6}(u)$ versus time corresponding to an orbit for which $\lambda=6.7675813202945$ is presented. Fig. 4(b) shows the approach of this orbit to the limit cycle by projecting it a 3-dimensional subvariety of phase space spanned by $\left(a_{3}, a_{7}, a_{4}\right)$. The effect of all modal coefficients acting together during the periodic can be seen by evolving the scalar field evaluated at the origin, $\phi(u, 0)=\lim _{r \rightarrow 0} \Phi(u, r) / r$ and shown in Fig. (c). In this case the period is $\Delta u \approx 0.05$.

A strong numerical indication that the limit cycle is not an artefact of the process of truncation is shown in Figs. 5, where (a) the limit cycle is projected in the plane $\left(a_{N-1}, a_{N}\right)$ for several values of $N$ and (b) the corresponding values of $\lambda_{*}$ is plotted in function of the truncation order $N$. 
As a matter of fact, these results suggest that the Galerkin method converges providing a reliable approach to the exact limit cycle as far as the truncation order is increased.

\section{Is the boundary fractal?}

Based on the above results the phase space contains two basins of attraction, namely, the subcritical and the supercritical basins for which the solutions tend, respectively, to the Minkowski spacetime and to form a black hole. The boundary between both basins is the critical solution represented by a limit cycle in phase space, and an interesting issue would be the determination if the basin boundary is fractal or not. In order to accomplish such a task we have applied a box counting method[17] with the uncertainty code defined by black hole/dispersion corresponding to the supercritical and subcritical basins, respectively. Briefly, the method consists in first, to choose a suitable set $\mathscr{D}$ of initial conditions close to the basin boundary. Second, let us consider a given initial condition $P\left(a_{k}(0)\right)$ belonging to $\mathscr{D}$, and perturb it by a very small radius $\varepsilon$ producing two other initial conditions schematically denoted by $P_{ \pm}\left(a_{k}(0) \pm \varepsilon\right)$. If the outcomes of $P$ and $P_{ \pm}$are distinct, then $P$ is considered an uncertain initial condition of $\mathscr{D}$; on the other hand if the outcomes are the same $P$ is a certain initial condition. Third, we plot the fraction $f$ of uncertain initial conditions versus the radius $\varepsilon$, for which the relation $f \propto \varepsilon^{\alpha}$ is satisfied, with $\alpha$ being the uncertainty exponent. It can be shown that if $\alpha=1$, then the basin boundary is smooth or not fractal; on the other hand for $0<\alpha<1$, the fractal dimension $d$ of the basin boundary is given by $d=D-\alpha$, where $D$ is the dimension of the phase space. In Fig. 6 the $\log$ - $\log$ plot of $f(\varepsilon)$ is depicted for the case $N=9$ and $J=1$, where we have considered $\varepsilon$ and for each small radius a set of 15,000 initial conditions was taken into account. The best fit of the linear region corresponds to $\alpha \approx 0.86$ indicating that the boundary is fractal.

\section{Further perspectives}

In this paper we have studied the spherical collapse of massless scalar field using the Galerkin method. The set of nonlinear partial differential equations (2.2), (2.3) and (2.4) was transformed into a finite set of ordinary differential equations or simply a dynamical system. We have discussed briefly the issue of accuracy by considering the linear problem, whose known exact solution provided the necessary test for the method. As an attractive feature of the method a relatively low truncation order of the Galerkin decomposition is able to attain a reasonable accuracy.

The next step consisted in considering the nonlinear problem described by the Einstein-KleinGordon equations. The resulting dynamical system dictates, no matter is the truncation order, the presence of three well defined classes of solutions determined by the initial strength of the scalar field. In fact this is in agreement with the previous analytical and numerical works. Using a dynamical system terminology the phase space is constituted by two basins of attraction: the subcritical basin for which the end configuration is the Minkowski spacetime, and the supercritical basin that indicated the formation of a black hole. The boundary between these two basins of attraction is the critical solution represented by a limit cycle in phase space.

Finally, as a consequence of this overall scenario the natural question of whether or not the boundary between both basins of attraction is fractal deserves more thoroughly investigation. As 
we have shown there is a strong indication that the boundary is fractal. Therefore, an interesting investigation would be naturally to increase the truncation order and improve the additional approximation (see for instance [18]), and to seek a relation between the fractal dimension and the critical exponent that appears in the scaling law of black hole formation (cf. (1.1)).

\section{Acknowledgments}

H. P. O. acknowledges CNPq/Brazil for partial financial support. The numerical experiments were made using Dynamics Solver package[19] and the $N$-dynamics package[20].

\section{References}

[1] Jeffrey Winicour, Characteristic Evolution and Matching, Living Rev. Relativity 8, (2005), 10. http://www.livingreviews.org/lrr-2005-10; Greg Cook, Initial Data for Numerical Relativity, Living Rev. Relativity 3, (2000), 5. http://www.livingreviews.org/lrr-2000-5.

[2] S. Bonazzola, E. Gourgoulhon and J. A. Marck, Spectral methods in general relativistic astrophysics, J. Comput. Appl. Math. 109, 433 (1999).

[3] Lawrence E. Kidder and lee samuel Finn, Spectral methods for numerical relativity: the initial data problem, Phys. Rev. D62, 084026 (2000).

[4] John P. Boyd, Chebyshev and Fourier Spectral Methods, 2001, Dover Publications, Inc.

[5] C. A. J. Fletcher, Computational Galerkin Methods, 1984, Springer-Verlag, New York Inc.

[6] Philip Holmes, John L. Lumley and Gal Berkooz, Turbulence, Coherent Structures, Dynamical Systems and Symmetry, 1998, Cambridge University Press.

[7] H. P. de Oliveira and I. Damião Soares, Preheating and Turbulence: Echoes of a not so Quiet Universe. Gen. Rel. Grav., 35, 2079 (2003). Third award of the Gravity Resarch Foundation competition 2003 ed.; H. P. de Oliveira and I. Damião Soares, Nonlinear Decay of the Inflaton: the Onset of Turbulence and Thermalization, JCAP (Journal of Cosmology and Astroparticle Physics), v.008, p.002 (2006).

[8] H. P. de Oliveira and I. Damião Soares, Gravitational wave emission from the numerical evolution of Robinson-Trautman spacetimes: A treatment in the nonlinear regime, Phys. Rev. D70, 84042 (2004).

[9] H. P. de Oliveira and I. Damião Soares, The dynamics of black hole formation: an evidence of nonextensivity, Phys. Rev. D71, 12403 (2005).

[10] H. P. de Oliveira and I. Damião Soares, Black Hole Bremsstrahlung: Can It Be an Efficient Source of Gravitational Waves?. Received the Honorable Mention in the Gravity Research Foundation competition 2006 ed.. To appear in Int. J. Mod. Phys. D (2006).

[11] H. P. de Oliveira and I. Damião Soares, Phys. Rev. D 65, 064029 (2002).

[12] M. W. Choptuik, Universality and scaling in gravitational collapse of a massless scalar field, Phys. Rev. Lett. 70, 9 (1993); "Critical" behavior in massless scalar field collapse, in Approaches to Numerical Relativity, p. 202, ed. R. d' Inverno, Cambridge University Press, 1992.

[13] C. Gundlach, Critical phenomena in gravitational collapse, Physics Reports 376, 339 (2003).

[14] Newman, E. T. and Penrose R., Proc. R. Soc. London Ser. A 305, 175 (1968). 
[15] Gómez, R., and Winicour, J., Asymptotics of Gravitational Collapse of Scalar Waves, J. Math. Phys., 33(4), 1445-1457, (1992).

[16] J. P. Boyd, J. Comp. Phys. 70, 63 (1987).

[17] Edward Ott, Chaos in Dynamical Systems, 1994, Cambridge University Press.

[18] H. P. de Oliveira, E. Rodrigues, I. D. Soares and E. V. Tonini, The low dimensional dynamical system approach in General Relativity: an example, in preparation.

[19] Juan M. Aguirregabiria, Dynamic Solver, http://tp.lc.ehu.es/jma/ds/ds.html. Copyright (C)1992-2006.

[20] Duarte, L. G. S., Mota, L. A. C. P., de Oliveira, H. P., Ramos, R. O., Skea, J. E. F., Numerical analysis of dynamical systems and the fractal dimension of boundaries, Comp. Phys. Comm., 199, 256 (1999). 


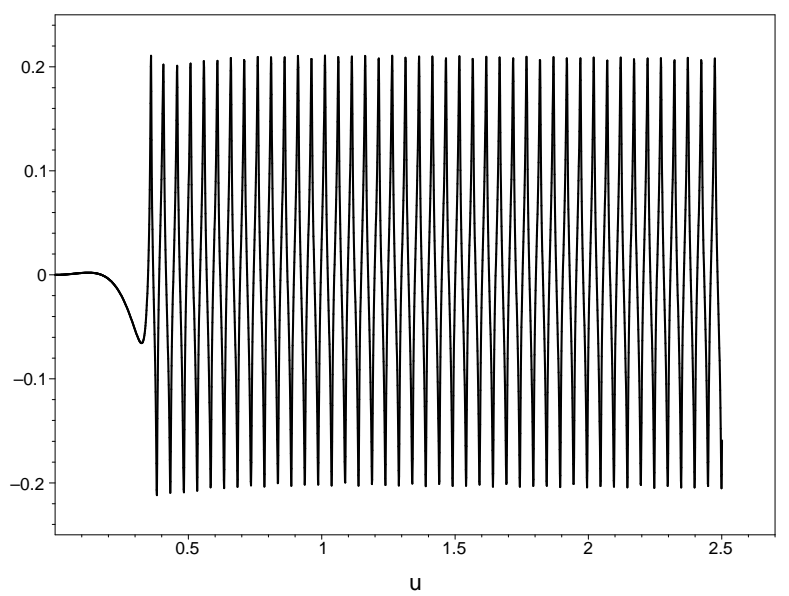

(a)

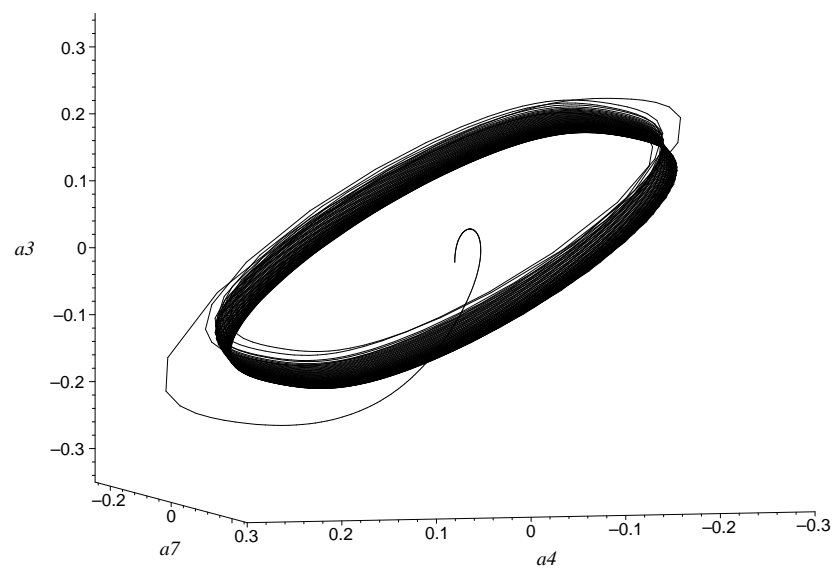

(b)

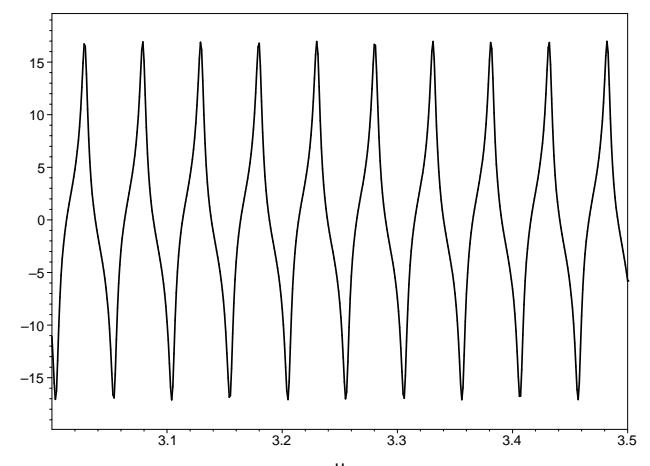

(c)

Figure 4: (a) Behavior of $a_{6}(u)$ for $\lambda \approx \lambda_{*}$. (b) Orbit approaching the limit cycle projected in the submanifold $\left(a_{3}, a_{4}, a_{7}\right)$ of phase space. (c) The scalar field at the origin $\phi(u, 0)$ for the (almost) critical solution. 

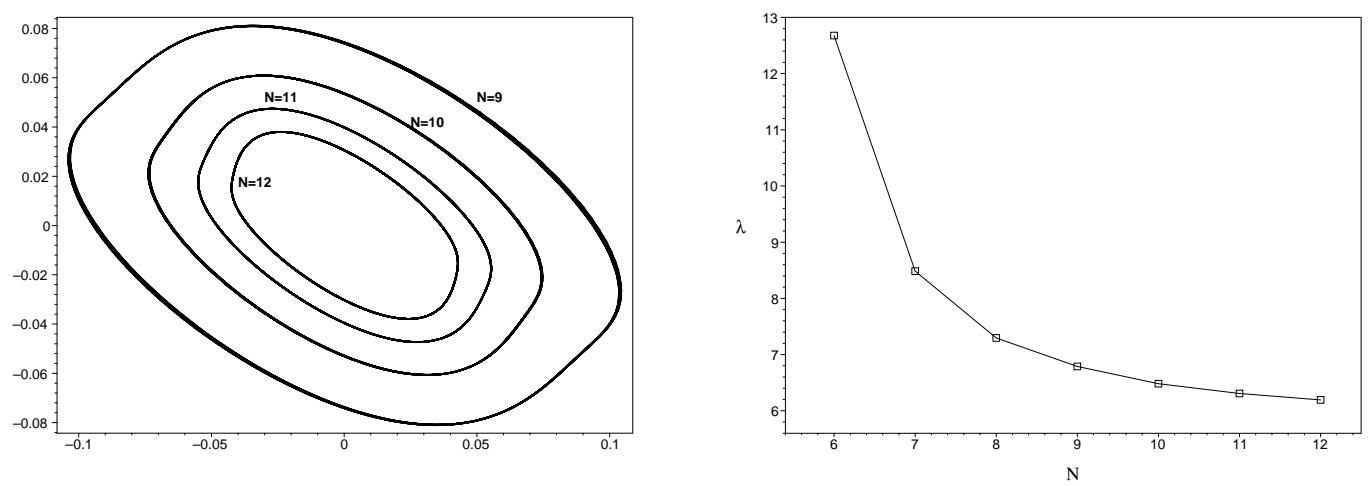

Figure 5: Projection of the limit cycle in the plane $a_{N-1}, a_{N}$ for the several truncation orders. The approach of the exact limit cycle is accomplished by increasing the truncation order. In the graph at right the critical values of $\lambda$ are plotted versus the truncation order indicating the convergence of the results.

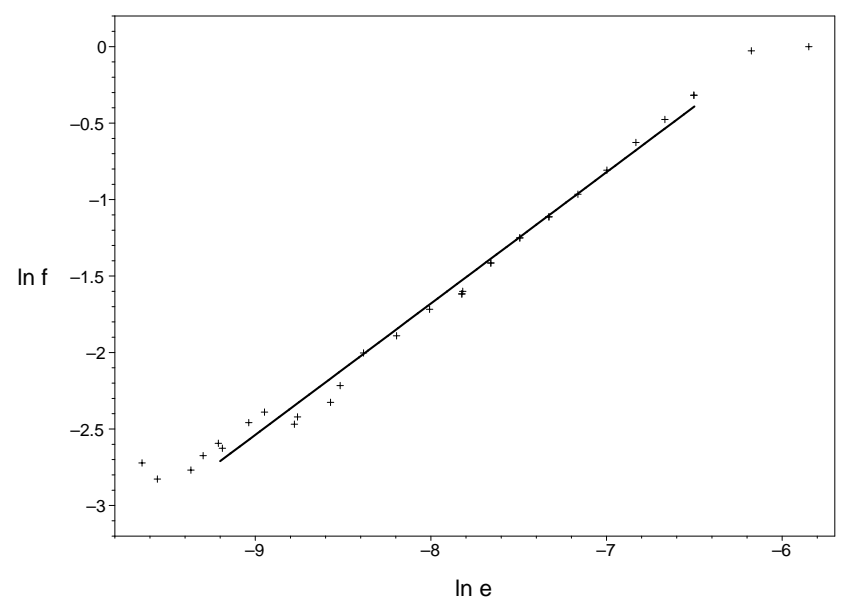

Figure 6: $\log -\log$ plot of the scaling law $f=f_{0} \varepsilon^{\alpha}$ for $N=9$ and $J=1$. 